\title{
Perceptual interference and facilitation with auditory imagery
}

\author{
MARTHA J. FARAH and ALBERT F. SMITH \\ Harvard University, Cambridge, Massachusetts
}

\begin{abstract}
It has been claimed both that (1) imagery selectively interferes with perception (because images can be confused with similar stimuli) and that (2) imagery selectively facilitates perception (because images recruit attention for similar stimuli). However, the evidence for these claims can be accounted for without postulating either image-caused confusions or attentional set. Interference could be caused by general and modality-specific capacity demands of imaging, and facilitation, by image-caused eye fixations. The experiment reported here simultaneously tested these two apparently conflicting claims about the effect of imagery on perception in a way that rules out these alternative explanations. Subjects participated in a two-alternative forced-choice auditory signal detection task in which the target signal was either the same frequency as an auditory image or a different frequency. The possible effects of confusion and attention were separated by varying the temporal relationship between the image and the observation intervals, since an image can only be confused with a simultaneous signal. We found selective facilitation (lower thresholds) for signals of the same frequency as the image relative to signals of a different frequency, implying attention recruitment; we found no selective interference, implying the absence of confusion. These results also imply that frequency information is represented in images in a form that can interact with perceptual representations.
\end{abstract}

What is the effect of imaging on the perception of stimuli? Two apparently contradictory answers to this question may be found in the literature. Segal and her colleagues (Segal, 1971; Segal \& Fusella, 1970; Segal \& Gordon, 1969) found evidence that images interfere with perception, so that external stimuli are less detectable to a subject who is imaging. Furthermore, Segal and Fusella (1970) found that this interference is selectively greater for stimuli that are similar to the image by virtue of being in the same modality: Auditory images lower $d^{\prime}$ (sensitivity) more for auditory stimuli than for visual stimuli, and visual images lower $\mathrm{d}^{\prime}$ more for visual stimuli than for auditory stimuli. Segal interpreted these findings in terms of confusions between the image and the stimulus: An imaging subject in a signal detection experiment must make a "reality decision" about the source of each perceptual experience. The more similar the image is to the stimulus, the harder that decision, resulting in lowered $\mathrm{d}^{\prime}$ values for the stimulus. It is possible, however, to explain Segal's results without assuming the confusability of images and signals. An alternative ex-

This work was supported by NSF Grant BNS 79-12418 awarded to Stephen M. Kosslyn. The authors are grateful to David $\mathbf{M}$. Green for the use of his laboratory equipment. The helpful comments of R. A. Finke, P. A. Jolicoeur, S. M. Kosslyn, R. D. Luce, and $R$. M. Nosofsky on an earlier draft of this paper are also gratefully acknowledged. The first author's mailing address is now: Psychology Service (116-B), Boston Veterans Administration Medical Center, 150 South Huntington Avenue, Boston, Massachusetts 02130 . planation based on the existence of modality-specific reserves of capacity or attention (see Shwartz, 1976) can account for Segal and Fusella's results if we assume that imaging in a particular modality uses some of that modality's capacity, leaving less modality-specific capacity available for encoding probe signals in that modality.

Another finding of imagery-based interference with signal detection comes from the work of Reeves (1981), who found interference between visual images and visual perception for chromatic, but not for achromatic, images and stimuli. Subjects who imaged an intensity increment of red light were less sensitive to actual intensity increments of red light against a white background than when they simply tried to detect the intensity increment without imaging, whereas subjects who imaged an intensity increment of white light were just as sensitive to an actual intensity increment of white light as when they were not imaging. Reeves also interprets his results by invoking confusion between images and signals, concluding that image-caused activation may be confused with externally caused activation in chromatic channels, but not in achromatic channels. Reeves's results could also be explained by capacity limitations, in this case by assuming that imaging chromatic stimuli requires more capacity than imaging achromatic stimuli. Because Reeves did not include conditions in which subjects imaged chromatic stimuli while detecting achromatic stimuli and vice versa, there is no way to distinguish between the confusion and capacity-limitation hypotheses. 
In contrast to these findings of interference between image and percept are the findings of Peterson and Graham (1974) that demonstrated facilitation of perception during imaging. Peterson and Graham had subjects study and then detect and name pictures of common objects. Two groups of subjects were used; both were cued during the detection and naming task with a name of a picture, but only one group received instructions to image the named picture. Correctly cued pictures were detected and named more accurately by the imaging subjects than by the nonimaging subjects, and incorrectly cued pictures were detected and named less accurately by imaging subjects than by nonimaging subjects. There was no difference between the two subject groups in ability to detect or name the pictures when no cue was given, which ruled out the possibility that the imaging group included more acute subjects.

Peterson and Graham interpreted their results in terms of the attention-recruiting capability of images. By preceding the perception of a stimulus with a compatible image, the subject has in effect "tailored" attention for the stimulus. However, it is possible that eye fixation, and not attention, is what has been altered by the image. Perhaps the imaging subjects fixed their eyes on the parts of the display screen that would contain the most salient parts of the cued picture.

These two sets of findings, increased perceptual interference and increased perceptual facilitation with more similar images, are not irreconcilable, and neither are their interpretations in terms of confusion and attention. Perhaps both image-caused confusion and attention operate together, and, depending on the experimental conditions, one or the other will dominate. The entire range of interference and facilitation effects (including Reeves's null effect for achromatic images and stimuli) could be accounted for in this way.

The experiment reported here was performed in order to establish whether or not interference due to confusion and facilitation due to attentional preparedness do operate together in a single experimental context when subjects perceive while imaging. In addition, the design of the present experiment precludes alternative explanations for these effects in terms of capacity limitations and eye fixation.

The predictions for this experiment are based on the assumption that an image can only be confused with a simultaneous stimulus, and not with a stimulus arriving later in time, whereas the effect of attentional priming can be enjoyed by a stimulus that follows immediately after the image or by a simultaneous stimulus if that stimulus is subthreshold and thus unable to draw attention to itself. If confusion alone operates, there should be selective interference between images and similar stimuli when the images are simultaneous with the stimulus presentations, but not when the images begin and end before the stim- ulus presentations. If attention alone operates, there should be selective facilitation for stimuli that are similar to images (relative to stimuli that are different) when the images are simultaneous with the stimulus presentations, and also when they begin and end before presentations. On the present hypothesis, that both attention and confusion operate, we ought to observe the combined effects of confusion and attention; the predicted pattern of data in this case is facilitation of perception of similar stimuli when a subject images before but not during a stimulus presentation, and either less facilitation or interference (depending upon the relative sizes of the confusion and attention effects) of perception of similar stimuli when a subject images during a stimulus presentation.

These predictions were tested in the context of an auditory signal detection task. The subjects imaged pure tones of one of two frequencies while performing a two-alternative forced-choice signal detection task in which one of the two frequencies was the target signal. In one session they imaged during the two observation intervals, and in a different session they imaged before the two observation intervals. The dependent measure was threshold intensity for detecting the signals. Note that this task requires only detection of the signal, not identification. If attentional facilitation operates in this experimental context, then thresholds would be lower for signals preceded by an image of the same frequency than by an image of a different frequency; if interference due to confusion also operates, then this effect would be diminished or reversed when the image occurred during the observation intervals.

By varying the similarity of the images and signals within one modality, we avoided the possibility that any selective interference between image and signal would be due to capacity limitations, rather than to confusions as hypothesized by Segal et al. and Reeves. We chose the auditory modality for three reasons. First, it provides a stimulus set, pure tones, in which a dimension of similarity (frequency) and a dimension of detectability (intensity) can be controlled independently and with great precision. Second, any facilitation we might observe could not be due to eye fixation. Third, we hoped to add to our knowledge of auditory imagery: If we were to find either selective interference or facilitation between images and signals of the same frequency, we would have established that frequency information is encoded in images, because it can affect the processing of auditory stimuli.

\section{METHOD}

\section{Procedure}

A two-alternative forced-choice detection procedure was used. In each trial, one of two signal frequencies was randomly selected for presentation, and signal intensity was varied adaptively according to a two-down, one-up "staircase" schedule. The adaptive 
steps were $5 \mathrm{~dB}$ prior to the fourth turnaround, and $1 \mathrm{~dB}$ thereafter. Signal levels at each turnaround after the fourth were averaged to yield an estimate of the signal level at which $71 \%$ of responses are correct. Through each block of 100 trials, the intensity of the two signals was varied according to independent, interleaved schedules. A continuous background noise of $60-\mathrm{dB}$ spectrum level was present throughout each block of trials. The starting intensity of each signal was $34 \mathrm{~dB}$ SPL. Each trial consisted of two 500 msec warning intervals, followed by two 500 -msec observation intervals. All intervals were indicated to the subjects by lights. The subjects responded by pressing one of two buttons labeled "first interval" and "second interval." Signals and noise were presented binaurally via TDH-50 headphones to subjects seated in doublewalled sound-treated chambers (IAC-1200 A).

The signals were generated by Wavetek oscillators and gated by electronic switches. The two frequencies used for the signals and the images were $715 \mathrm{~Hz}$ and $1000 \mathrm{~Hz}$. The signal duration was $500 \mathrm{msec}$, completely filling the observation interval. The timing of the experiment, adaptive attenuation of signals, and data storage were controlled by a PDP-15 computer.

The subjects were instructed to image tones of one of the two signal frequencies in one set of-specified intervals throughout a block of 100 trials. In the "image-before" condition, the subjects imaged two tones during the two warning intervals. In the "imageduring" condition, the subjects imaged two tones during the two observation intervals. Pilot work revealed that when the subjects were in the "image-before" condition, they tended to adopt a strategy of ending their image by briefly imaging a different tone. We therefore emphasized that the subjects should image only the required tone, and only during the required interval. Before each block of 100 trials, the subjects were allowed to hear the tone to be imaged and to practice imaging it. A few seconds after they said that they were ready, a block of trials began. Every 20 trials, the subjects were given eight repetitions of the tone to be imaged, after which they corrected their image if it was off-key, and cleared their throats and said the word "hello" aloud (so that any short-lived perceptual effects of hearing the to-be-imaged tone would not affect their signal detection performance).

\section{Design}

The experiment occurred in two sessions of four blocks of 100 trials each. For half of the subjects, the first session consisted of the image-before condition and the second session consisted of the image-during condition, and for the other half, this order was reversed. Half of the subjects within each of these groups imaged the higher frequency in the first and fourth blocks of trials and the lower frequency in the second and third blocks of trials, and half imaged the lower frequency first and fourth and the higher frequency second and third.

\section{Subjects}

Eight Harvard/Radcliffe undergraduates served as subjects for pay. An additional three subjects were not used because they informed the experimenters after the first experimental session that they were unable to form auditory images more than $60 \%$ of the time.

\section{RESULTS}

Estimates of the threshold of each signal frequency in each block of trials for each subject were submitted to a repeated-measures analysis of variance, in which the factors were location of image (beforeduring), image frequency $(715-1000 \mathrm{~Hz}$ ), signal frequency $(715-1000 \mathrm{~Hz})$, and part of experimental session (first half-second half).

The eight mean thresholds of interest are plotted in Figure 1. It is immediately apparent that there is an overall tendency for selective facilitation, that is, that

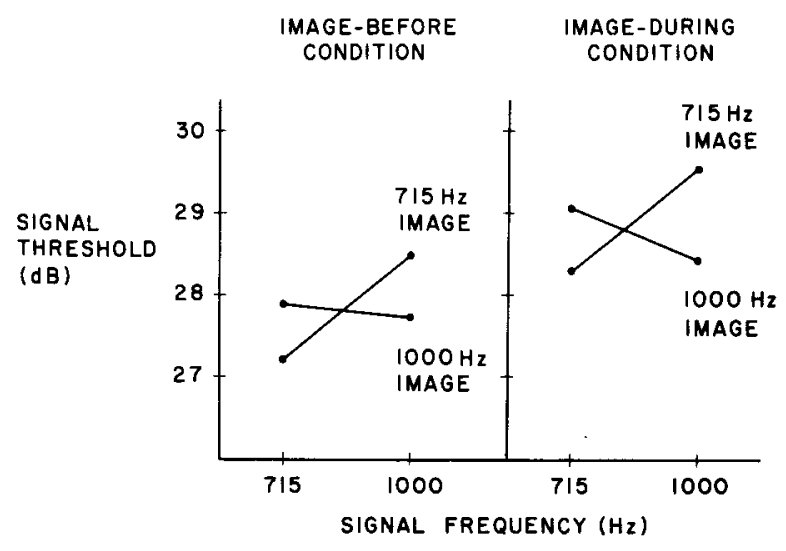

Figure 1. Threshold (in decibels) for detecting signals. Left panel: Image precedes observation intervals. Right panel: Image coincides with observation intervals.

thresholds were lower when image and signal frequencies were the same, and this interaction between image and signal frequency is significant $[F(1,7)=$ $8.13, p<.05]$. The mean thresholds for the 715 and $1000-\mathrm{Hz}$ signals were 27.77 and $29.03 \mathrm{~dB}$, respectively, with a $715-\mathrm{Hz}$ image, and 28.51 and $28.10 \mathrm{~dB}$, respectively, with a $1000-\mathrm{Hz}$ image.

Also clearly visible in Figure 1 is the significant main effect of when, in the course of a trial, an image was formed and held $[F(1,7)=8.42, p<.05]$. The mean threshold for the image-before condition was $27.85 \mathrm{~dB}$, and the mean threshold for the imageduring condition was $28.85 \mathrm{~dB}$. This nonselective interference is presumably a measure of the effort drawn away from the signal detection task by the activity of forming and holding an image.

The difference in the amount of facilitation between the image-before and image-during conditions (the interaction among image location, image frequency, and signal frequency), which is crucial for establishing the existence of selective interference, is not significant $(\mathrm{F}<1)$. The only other significant effect is an interaction between the image frequency and the half of the experimental session in which the image occurred $[F(1,7)=7.8, p<.05]$. During the first half of the experimental sessions, the mean signal thresholds with 715 - and $1000-\mathrm{Hz}$ images were 28.78 and $28.23 \mathrm{~dB}$, respectively, and during the second half of the experimental sessions, they were, in the same order, 28.01 and $28.38 \mathrm{~dB}$. This unexpected interaction could reflect different rates of familiarization and fatigue for the two image frequencies. No other effects were significant $(p>.1)$.

\section{DISCUSSION}

The principle findings of this experiment can be summarized as follows.

First, the subjective frequency of an auditory image affects perceptual processing, which implies that frequency information is represented in images. 
Lest this result seem so obvious from introspection as to be uninteresting, we note the recent finding of Intons-Peterson (1980) that loudness is not necessarily represented in auditory images. Surprisingly, her subjects' images did not include a dimension corresponding to the loudness of the sounds that they were imaging unless the task for which imagery was used explicitly required manipulation or comparison of image loudness.

Second, images facilitate detection of samefrequency signals relative to different-frequency signals. In other words, images cause a change in the allocation of available processing capacity that favors the encoding of same-frequency images over different-frequency images. This finding is consonant with the findings of Peterson and Graham (1974) with visual imagery and implies that the attentional facilitation found in their experiment reflects a general property of imagery and attention, rather than a peculiarity of visual processing or an artifact such as eye fixation. We believe that the nature of the relationship between imagery and attention deserves further examination. One possibility is that by generating an image, a subject in effect supplies his or her own "stimulus" to drive the automatic, stimulus-driven process of attention. Another possibility is that imagery and attention are at some level of analysis the same process, a view held by Neisser (1976).

Third, the selective facilitation was no greater in the image-before condition than in the image-during condition, which implies that interference effects due to confusions between images and signals were either nonexistent or much smaller than the facilitation effects found in this experiment.

This failure to find selective interference surprised us, because the majority of the subjects in this experiment reported experiencing confusions. Several subjects were quite insistent that we were presenting more than one signal per trial, and, significantly, when those subjects were in the image-before condition they claimed that the extra signals were occurring outside the observation intervals! Why then did these confusions have no measurable effect on the subjects' signal detection performance? The subjects' introspections suggest an interesting answer. The subjects who reported vivid "false alarms" from imaging during the observation intervals tended to treat the signal detection task as a discrimination between one signal and either two signals or one louder signal. When viewed this way, it is clear why imagecaused confusions should have only a small effect on signal detection performance: To the extent that the image has intensity, it will have the effect of raising the background intensity, or "pedestal," against which the signal is detected as an intensity increment. By Weber's law, this will make the signal detection task only gradually harder as the image intensity increases. Put another way, the "reality decision" mentioned earlier must in fact be a choice between signal plus image plus noise and image plus noise, not the harder discrimination between signal plus noise and image plus noise.

This analysis of image-caused interference suggests that, if selective interference exists, it is a very small effect. This is consistent with the fact that we found no selective interference, despite our ability to detect both selective facilitation and nonselective interference caused by images with our experimental paradigm. It also casts doubt on Segal et al.'s claims that the interference observed in their experiments was due to image-signal confusions, rather than to general and modality-specific capacity limitations.

To the question posed at the beginning of this paper, then, we have two answers: First, we found evidence that imagery facilitates perception by means of a central attentional mechanism, as Peterson and Graham (1974) claimed. Second, we found that the selective interference caused by confusing images and signals, if it exists at all, is a small effect compared with the facilitatory effect of imagery on perception. Furthermore, we argued that this second finding is to be expected, by Weber's law, because image-caused activation will add to both the signal and the noise activation in perceptual channels.

\section{REFERENCES}

Intons-Peterson, M. J. The role of loudness in auditory imagery. Memory \& Cognition, 1980, 8, 385-393.

Neisser, U. Cognition and reality. San Francisco: Freeman, 1976.

Petghson, M. J., \& Grakam, S. E. Visual detection and visual imagery. Journal of Experimental Psychology, 1974, 103, 509-514.

REEvEs, A. Visual imagery lowers sensitivity to hue-varying, but not luminance-varying, visual stimuli. Perception \& Psychophysics, 1981, 29, 247-250.

Segal, S. J. Processing of the stimulus in imagery and perception. In S. J. Segal (Ed.), Imagery: Current cognitive approaches. New York: Academic Press, 1971.

SEGal, S. J., \& Fusella, V. Influence of imaged pictures and sounds on the detection of visual and auditory signals. Journal of Experimental Psychology, 1970, 83, 458-464.

Segal, S. J., \& Gordon, P. The Perkey effect revisited: Paradoxical threshold or signal detection error. Perceptual and Motor Skills, 1969, 28, 791-797.

Shwartz, S. P. Capacity limitations in human information processing. Memory \& Cognition, 1976, 4, 763-768.

(Manuscript received June 1, 1982; revision accepted for publication December 27, 1982.) 